CONFORMAL GEOMETRY AND DYNAMICS

An Electronic Journal of the American Mathematical Society

Volume 4, Pages 97-107 (August 23, 2000)

S $1088-4173(00) 00054-0$

\title{
MATRIX REPRESENTATIONS AND THE TEICHMÜLLER SPACE OF THE TWICE PUNCTURED TORUS
}

\author{
J. O. BUTTON
}

\begin{abstract}
We realise the Teichmüller space of the twice-punctured torus as a set of triples of matrices that are suitably normalised. As a consequence, we see the space as a simple open subset of $\mathbb{R}^{4}$ which is obtained directly from the matrix entries. We also discuss the connection between this representation and the one in terms of the traces of elements.
\end{abstract}

\section{IntRoduction}

The Teichmüller space of a surface is an important object, and as evidence of its importance we see in the literature many definitions, seemingly different but all ultimately the same. For instance, topologists would define it in terms of diffeomorphisms isotopic to the identity (see [19]), and complex analysts would define it in terms of quasi-conformal mappings (see [1). However we do this though, as a topological space it is homeomorphic to $\mathbb{R}^{n}$ for the appropriate value of $n$, and a lot of effort goes into parametrising it, namely embedding it into $\mathbb{R}^{n}$ as a connected open subset, where the coordinates of a point in Teichmüller space can be suitably interpreted in terms of the original definition. Of course different definitions will yield different subsets, and there will be variations depending on what properties we wish this embedding to have. Many approaches are based on considering hyperbolic structures on the surface, along with a marking of the fundamental group. This is the approach taken by Bers (see 2]) where the points are bounded quadratic differentials, and by Thurston using projective measured laminations (see [6]), and in both these cases Teichmüller space will be embedded into $\mathbb{R}^{n}$ as a bounded domain, thus giving rise to a compactification which allows interpretation of points on the boundary. Another approach using the hyperbolic structure is to find parameters that relate to the surface as closely as possible, for instance if the surface has a hyperbolic structure, then we can use the lengths of certain closed geodesics and twisting angles. See 7 for this approach.

However, in this paper we take the view that a surface with a hyperbolic structure is given to us concretely by a Fuchsian subgroup, namely the discrete subgroup of $\operatorname{PSL}(2, \mathbb{R})$ by which we quotient the upper half plane to obtain this structure, which is determined by this subgroup up to conjugacy. Thus to study the group is to study the surface, and we use an equivalent definition of Teichmüller space as isomorphisms of a base group into $P S L(2, \mathbb{R})$ up to conjugacy that preserve parabolic elements and whose image is discrete. This embeds naturally into the space of all representations of the base group into $\operatorname{PSL}(2, \mathbb{R})$ which preserve parabolics.

Received by the editors August 16, 1999 and, in revised form, July 10, 2000.

2000 Mathematics Subject Classification. Primary 20H10; Secondary 32G15. 
We wish to find coordinates which are as simple as possible for the Teichmüller space, and there are two related approaches. One is to choose from each conjugacy class of $k$-tuples of matrices (where $k$ is the size of a minimal generating set for the group) a particular representative $k$-tuple, by normalising the matrices in some preferred fashion, so that there will be one point in this space for each conjugacy class of isomorphisms. Thus we can think of the parameters in the matrices as the representation space and then find out which subset corresponds to the homomorphisms whose image is discrete and faithful. The other is to use the traces of certain elements of the group, which will be the same for any conjugate group. If we take enough traces, so that the point obtained defines a group uniquely up to conjugation, then this can also be used as the representation space. These traces are sometimes called Fricke moduli (see 8]).

In the literature, there are many examples of coordinates for the Teichmüller space of a surface based on its uniformisation by Fuchsian or Kleinian groups. In 11 the Teichmüller space $T_{g}$ of a closed surface of genus $g$ is biholomorphically embedded in $\mathbb{C}^{3 g-3}$, where the parameters are functions of traces and of cross ratios of elements of a Kleinian group having a simply connected invariant component of its region of discontinuity whose quotient by the group gives us the hyperbolic structure on the surface. This approach is followed up in [5] where again $T_{g}$ is biholomorphically embedded in $\mathbb{C}^{3 g-3}$, this time using uniformisation by quasiFuchsian groups. At the same time, the paper of Bers [3] (which also deals with biholomorphic embeddings of Teichmüller spaces with punctures) provides a summary of the purpose of this work when he states "the main aim of Teichmüller theory is to make the dependence of a Riemann surface (of finite type) on the complex or real moduli as explicit and as transparent as possible". Also in that year, the paper of Kra and Maskit considers quasiconformal deformations of Kleinian groups, and it uses coordinates defined in terms of the images of points in $\mathbb{C}$ under a normalised deformation. In [17] the Teichmüller space $T_{g}$ is parametrised using multipliers of elements of a Fuchsian group uniformising the surface, and the proof is based on the signs of the traces of certain elements, and the separation properties of their axes that are consequent. Subsequently these parameters are converted into geodesic lengths in [18. In [9] complex embeddings of $T_{g}$ are again discussed, using the plumbing construction.

As for explicit coordinates for particular Teichmüller spaces of low dimension, one is particularly interested in embeddings where the exact region corresponding to Teichmüller space is known and is, hopefully, as simple as possible. In the same book as 18, is a paper by Maskit 12] where coordinates are provided for the Teichmüller space of a four punctured sphere. They are in terms of two parameters $x$ and $y$ with $x>1$ and $y<0$, which are obtained by cutting up the surface. Normalised matrices generating the Fuchsian group uniformising the surface are also given in terms of $x$ and $y$. There are also in the literature numerous parametrisations of the Teichmüller space of the once-punctured torus (such as [13]), which similarly can be embedded into $\mathbb{R}^{2}$. As for the simplest possible compact hyperbolic surface, of genus 2, we find in [14] explicit matrices for Fuchsian groups uniformising this surface, written in terms of parameters for $T_{2}$ which are defined in terms of fixed points of elements of the group. Very recently, a paper by the same author [15] introduces new parameters for $T_{2}$ which give rise to a real analytic embedding of $T_{2}$ into $\mathbb{R}^{6}$ where the region is explicitly defined, in terms of three trivial and two non-trivial inequalities (a trivial inequality is where the sign of a parameter is asserted). The 
matrices forming the marked generating set of the Fuchsian group uniformising the surface are presented in terms of these parameters, which themselves are functions of lengths of simple closed geodesics and angles and distances between simple closed geodesics on the surface. That publication is very close to the aims and results of this paper, where we examine Teichmüller space in the case where the surface is a twice-punctured torus. After giving necessary and sufficient conditions for a representation to be discrete and faithful in section 1 , we then obtain a particularly neat normalisation in section 2. The fact that the matrices obtained have entries which are simple functions of the parameters being used is pleasing, but this is not its main strength. The power of the normalisation is that we can easily find the exact subset of the representation space which is equal to Teichmüller space (it is given by four trivial inequalities and one non-trivial inequality), so that we can tell by a glance at these matrices whether the group generated by them is discrete with quotient surface a twice-punctured torus or not. The reason why the correct subspace can be easily found without having to check whether all groups contained in it are discrete (which would mean having to find an appropriate fundamental domain for each group, or some other discreteness criterion) is that the Teichmüller space of a surface of finite hyperbolic area is a connected component of the representation space. The process can be thought of heuristically in the following way: the entries of the generating matrices for the group are rational functions of certain parameters, and these parameters make up the representation space. If we start with parameter values which are known to generate a twice-punctured torus group, then we can vary all parameters whilst still remaining in Teichmüller space, because it is an open subset. However there are no points in the representation space lying on the boundary of Teichmüller space. Thus as we move in Teichmüller space further and further away from our original point, we never reach the "edge" but find that a matrix entry in our normalisation has "blown up", in that the denominator is tending to zero. However, if we take parameter values for which the denominator is equal to zero, then our normalisation has broken down, and so this is not a point of the representation space. Finally, in section 3 we compare this approach to that where traces are used as parameters for the representation space and Teichmüller space.

\section{Discrete faithful Representations}

The twice-punctured torus is a surface whose fundamental group is the free group on three generators, and which has two conjugacy classes of peripheral curves. It can be represented as a quotient of the upper half plane $U$ by a Fuchsian group $G$ whose limit set is the whole of the real line, thus obtaining a complete hyperbolic surface of finite area. Taking a particular $G_{0}$ as a base surface, we will have $G_{0}=\langle A, B, C\rangle$ where $A, B, C \in P S L(2, \mathbb{R})$ freely generate $G_{0}$, and we can take two words $w_{1}, w_{2}$ in $A, B, C$ which are parabolic and which are a full list of parabolics; i.e. every parabolic element of $G_{0}$ is a power or a conjugate of a power of $w_{1}$ or $w_{2}$. We can define (see 4]) the Teichmüller space $\mathcal{T}$ of the twice-punctured torus to be the set of isomorphisms of $G_{0}$ into $P S L(2, \mathbb{R})$ up to conjugacy in $P G L(2, \mathbb{R})$ whose image is discrete, and which are type-preserving, namely that the image of any parabolic element is parabolic (or the identity). As it stands, this definition of Teichmüller space depends on $G_{0}$ but is independent of any generating set for $G_{0}$, and of the parabolic words $w_{1}, w_{2}$. However, when we put a topology on $\mathcal{T}$ we now fix a 


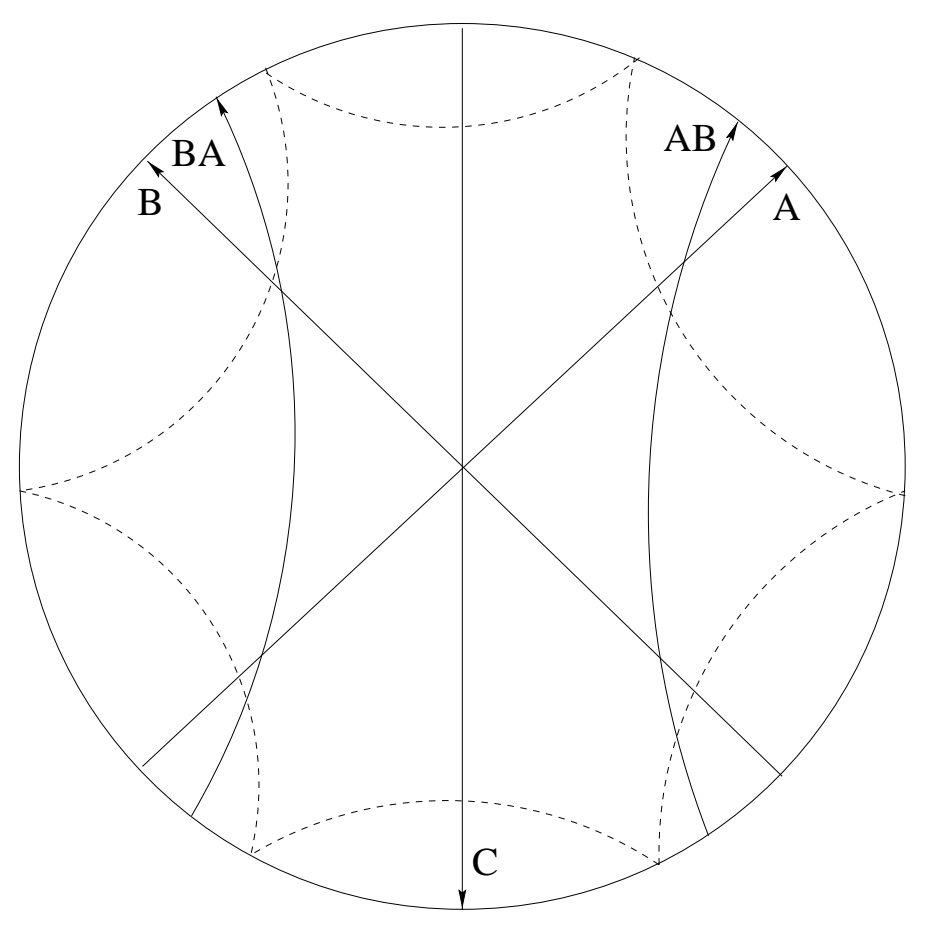

FiguRE 1.

generating set $\left(A_{0}, B_{0}, C_{0}\right)$ for $G_{0}$, along with the words $w_{1}, w_{2}$, and we embed $\mathcal{T}$ into $P S L(2, \mathbb{R})^{3} / P G L(2, \mathbb{R})$ where $P G L(2, \mathbb{R})$ acts on $P S L(2, \mathbb{R})^{3}$ by simultaneous conjugation. We can then think of $\mathcal{T}$ as a subset of the representation space $\mathcal{R}$, where

$$
\mathcal{R}=\left\{[(A, B, C)] \in P S L(2, \mathbb{R})^{3} / P G L(2, \mathbb{R}):\left|\operatorname{tr}\left(w_{1}\right)\right|=\left|\operatorname{tr}\left(w_{2}\right)\right|=2\right\},
$$

thus in this realisation of $\mathcal{T}$ one can think of the identity isomorphism as now being the equivalence class of points $\left[\left(A_{0}, B_{0}, C_{0}\right)\right]$ in $\mathcal{R}$.

As $G_{0}$ is a free group on three generators, it is completely canonical (and would be strange otherwise) to use generating sets consisting of a triple of elements freely generating $G_{0}$. However, there is no such canonical choice for the words $w_{1}$ and $w_{2}$. In this paper, we proceed by choosing a $G_{0}$ which has a six sided fundamental domain for its action on the upper half plane $U$. This fundamental domain is shown in figure 1, except that in order to picture it more clearly we have conjugated $G_{0}$ using a Möbius transformation that sends $U$ to the unit disc $D$. Thus the conjugate of $G_{0}$ appearing in figure 1 is not a subgroup of $P S L(2, \mathbb{R})$, but a discrete group of Möbius transformations that preserves $D$. However, throughout the text all groups mentioned are subgroups of $P S L(2, \mathbb{R})$. The generators have axes which intersect pairwise, and which are oriented as in the diagram. There are three pairs of arcs represented by the dotted lines, with one in each pair being mapped by a generator to the other one. The hexagonal region bounded by the six arcs can be taken as a fundamental domain for $G_{0}$ provided that the six vertices are each stabilised by parabolic elements. We see from figure 1 that those stabilisers are $A B C, C B A$ and 
their cyclically reduced conjugates. Performing the identifications, we see that we do indeed obtain a twice-punctured torus.

Therefore throughout this paper we take as our parabolic elements the words $w_{1}=A B C$ and $w_{2}=C B A$, up to conjugacy. It is no problem if others choose different words for the parabolic elements, as we can then use an automorphism to change from one set of words to the other (or possibly their inverses). For instance, a presentation including parabolic words for the twice-punctured torus which is often given is

$$
G=\left\langle X, Y, P_{1}, P_{2}: w_{1}=P_{1}, w_{2}=P_{2} ; X Y X^{-1} Y^{-1} P_{1} P_{2}\right\rangle
$$

which, as a free group, is

$$
G=\left\langle X, Y, Z: w_{1}=Z, w_{2}=Z^{-1} Y X Y^{-1} X^{-1}\right\rangle
$$

but we get back to our presentation by setting $X=B, Y=A, Z=A B C$ and replacing $w_{2}$ with its inverse.

We wish to find a convenient form for $\mathcal{T}$, which can be obtained either by parametrising matrices which are suitably normalised, or by using the traces of certain elements. We take the first approach in the next section, and then move onto traces in section 3 . Note that the space $\mathcal{T}$ is of four real dimensions; this can be seen quickly by the fact that $\operatorname{PSL}(2, \mathbb{R})^{3}$ is a nine dimensional space, which is then reduced by three owing to the conjugation action of $P G L(2, \mathbb{R})$, and then by a further two because of the conditions set on the two traces. However, we will be using the traces of six primitive words of length one or two in the generators, thus we set

$$
(a, b, c, x, y, z)=(\operatorname{tr}(A), \operatorname{tr}(B), \operatorname{tr}(C), \operatorname{tr}(B C), \operatorname{tr}(C A), \operatorname{tr}(A B))
$$

which is a point in $\mathbb{R}^{6}$. As the trace of an element in $\operatorname{PSL}(2, \mathbb{R})$ is only defined up to sign, we are free to choose the signs of $a, b, c$ independently. Once we have done this though, the signs of $x, y, z$ are determined. Also note that for our group $G_{0}$ in figure 1, the commutator $A B A^{-1} B^{-1}$ is a hyperbolic element of $\operatorname{PSL}(2, \mathbb{R})$ with a trace that has a well defined sign (independent of the signs chosen for the traces of $A$ and $B$ ), which is equal to $a^{2}+b^{2}+z^{2}-a b z-2$. But because the axes of $A$ and $B$ intersect, this means that it has a negative sign, so that $a^{2}+b^{2}+z^{2}-a b z<0$. Similarly, we reach the same conclusion about the other two commutators. Also we know that if we take $a, b, c>2$, then $x, y, z>2$ as well, because each pair of generators has intersecting axes. If we now look at the pair of elements $A B$ and $C$, we see that they have non-intersecting axes but that the parabolic fixed point $A B C$ is not separated by one axis from the other. This means (see [4]) that the trace of $A B C$ must be negative, so that it equals -2 . In the same way, the location of the axes of $C$ and $B A$ tells us that $\operatorname{tr}(C B A)=-2$ as well. In fact, having chosen our parabolic words as above, these properties of the signs of the traces characterise twice-punctured torus groups.

Theorem 1.1. If $(A, B, C) \in P S L(2, \mathbb{R})^{3}$ and $A B C, C B A$ have $\mid$ trace $\mid=2$, then $G=\langle A, B, C\rangle$ is a discrete group with its quotient surface equal to a twice-punctured torus if and only if (writing $A, B, C$ as matrices with positive traces) we have

$$
\begin{aligned}
& a^{2}+b^{2}+z^{2}-a b z<0, \\
& b^{2}+c^{2}+x^{2}-b c x<0, \\
& c^{2}+a^{2}+y^{2}-c a y<0,
\end{aligned}
$$


and $\operatorname{tr}(A B C)=\operatorname{tr}(C B A)=-2$, where $(a, b, c, x, y, z)$ is as above.

Proof. If the above conditions are satisfied on the traces, then the information provided is exactly enough to be able to place the axes and we find that $G$ is a Schottky group, except that the two peripheral elements are parabolic, thus we have satisfied the conditions for Poincare's polyhedron theorem. See [4] for details of the argument.

Now suppose that the quotient of the upper half plane (or hyperbolic disc) by $G$ is topologically a twice-punctured torus, so that $G$ is the fundamental group of this surface. We can define the obvious isomorphism from $G_{0}$ to $G$ by forgetting the zero subscripts. This will be a type-preserving isomorphism, so that its equivalence class lies in $\mathcal{T}$. This means that the isomorphism is induced by a quasi-conformal homeomorphism from the quotient surface of $G_{0}$ to that of $G$, which can be lifted to a quasi-conformal homeomorphism of the upper half plane, and then extended to the Riemann sphere. This must preserve the locations of the fixed points of words in $A, B, C$ (or reverse them if the homeomorphism is orientation reversing) so that the argument applied above to $G_{0}$ works equally well for $G$.

Thus if we can show that this sextuple defines the ordered triple of matrices $(A, B, C)$ uniquely up to simultaneous conjugation, then we can think of $\mathcal{T}$ as a subset of $\mathbb{R}^{6}$. We will show that this is indeed the case in section 2. However, this is not useful in practice without further details; for instance if we try to find a particular sextuple representing a group in $\mathcal{T}$, then we would first need to find six real numbers such that the matrices obtained give rise to $A B C$ and $C B A$ which are both parabolic with trace -2 . This means that we will have two equations (given in section 3 ) in these six variables to solve. We would then have to ensure that the inequalities in Theorem 1.1 are satisfied. Moreover, suppose we wish to find sextuples where all entries are rational, or lie in ones favourite number field, then choosing four elements of the sextuple to lie in this field does not guarantee that the remaining pair of elements found by solving the two equations will do so as well. Also, as $\mathcal{T}$ is four dimensional, we really want to see it as a subset of $\mathbb{R}^{4}$. This we do in the next section by finding representative matrices for $A, B, C$.

\section{MATRIX REPRESENTATIONS}

Given any twice-punctured torus group $G=\langle A, B, C\rangle$, we now want to find the explicit matrices $A, B, C$ where $A B C$ and $C B A$ are parabolic with trace equal to -2 , so that $G$ has a fundamental domain just like that in figure 1 . The idea is to normalise so that $A B C$ fixes $\infty$ and the conjugate element $B C A$ fixes 0 , thus obtaining

$$
A B C=\left(\begin{array}{rr}
-1 & -j \\
0 & -1
\end{array}\right), \quad B C A=\left(\begin{array}{rr}
-1 & 0 \\
k & -1
\end{array}\right) \quad \text { for } j, k \in \mathbb{R}-\{0\} .
$$

We will show that we can take $j=k>0$. Note that the signs of $j$ and $k$ will be the same, because $B C A$ has to be a conjugate of $A B C$ by an element of $P S L(2, \mathbb{R})$. We can then further conjugate by $z \mapsto \lambda z$ (which will be in $P S L(2, \mathbb{R})$ if $\lambda>0$ and in $\operatorname{PGL}(2, \mathbb{R})-\operatorname{PSL}(2, \mathbb{R})$ if $\lambda<0)$ to ensure that $j=k>0$.

This means that $A$ and $B C$ will be of the following form:

$$
A=\left(\begin{array}{rr}
a & -1 \\
1 & 0
\end{array}\right) \quad \text { and } \quad B C=\left(\begin{array}{rr}
0 & -1 \\
1 & x
\end{array}\right)
$$


with $k=a+x$ (where we are making a choice from one of the two matrices in $S L(2, \mathbb{R})$ representing the transformation $A$ by insisting that the entry in the bottom right hand corner is 1 ).

We then set

$$
B=\left(\begin{array}{cc}
\alpha & \theta \\
\frac{b \alpha-\alpha^{2}-1}{\theta} & b-\alpha
\end{array}\right) \quad \text { and } \quad C=\left(\begin{array}{cc}
\delta & \zeta \\
\frac{c \delta-\delta^{2}-1}{\zeta} & c-\delta
\end{array}\right)
$$

where we know that no entries in $B$ or $C$ are zero, because otherwise they would either share a fixed point with $A B C$ or $B C A$ (which would mean that the group generated would not be discrete, or would not be free on three generators), or one of $B^{ \pm 1}, C^{ \pm 1}$ would send $\infty$ to 0 , and then $A B^{ \pm 1}$ or $A C^{ \pm 1}$ would share a fixed point with $B C A$, thus obtaining a contradiction as before.

Comparing our two different expressions for $B C$, we first evaluate the zero term, which enables us to eliminate $\zeta=\theta\left(1+\delta^{2}-c \delta\right) / \alpha \delta$. Then by doing the same with the 1 term, we obtain $\theta=-\delta$. Finally the $x$ term gives us the relation $\alpha \delta x=b \alpha+c \delta-\alpha^{2}-\delta^{2}-1$. By using this expression for $x$, we can also find the traces $y=\delta(x+a)-b$ and $z=\alpha(x+a)-c$.

We now have our matrices depending on five real variables $a, \alpha, \delta, b, c$, but we have not used the fact that $\operatorname{tr}(C B A)$ must be -2 . In order to make the calculations more straightforward, we set $\beta=\alpha b-1-\alpha^{2}$ and $\gamma=\delta c-1-\delta^{2}$. Thus now

$$
B=\left(\begin{array}{rr}
\alpha & -\delta \\
-\frac{\beta}{\delta} & b-\alpha
\end{array}\right) \quad \text { and } \quad C=\left(\begin{array}{cc}
\delta & \frac{\gamma}{\alpha} \\
\alpha & c-\delta
\end{array}\right) .
$$

We evaluate $\operatorname{tr}(C B A)$ which must equal -2 , and obtain

$$
\alpha \delta\left(\alpha^{2} \delta^{2}-\beta \gamma\right) a=\alpha^{2} \delta^{2}\left(\alpha^{2}+\delta^{2}-2\right)-\beta \gamma\left(\alpha^{2}+\delta^{2}\right)-\beta \alpha^{2}-\gamma \delta^{2}
$$

where we have now replaced all remaining appearances of $b$ and $c$ with $\beta$ and $\gamma$. Thus we obtain the following:

Theorem 2.1. Given a point $p \in \mathcal{T}$, there exists a unique triple $(A, B, C)$ of elements of $P S L(2, \mathbb{R})$ in the equivalence class of $p$ which can be written as matrices in the form

$$
A=\left(\begin{array}{rr}
a & -1 \\
1 & 0
\end{array}\right), \quad B=\left(\begin{array}{rr}
\alpha & -\delta \\
-\frac{\beta}{\delta} & \frac{\beta+1}{\alpha}
\end{array}\right) \quad \text { and } \quad C=\left(\begin{array}{cc}
\delta & \frac{\gamma}{\alpha} \\
\alpha & \frac{\gamma+1}{\delta}
\end{array}\right)
$$

for $\alpha, \delta>0$ and non-zero $\beta, \gamma$, with a satisfying (2.1). Alternatively, taking any $\alpha, \delta>0$ and $\beta, \gamma \neq 0$, if $\alpha^{2} \delta^{2} \neq \beta \gamma$ then we can form matrices $(A, B, C)$, giving rise to a triple of elements of $P S L(2, \mathbb{R})$ such that $\operatorname{tr}(A B C)=\operatorname{tr}(C B A)=-2$.

Proof. Given $p \in \mathcal{T}$, we follow the steps of the normalisation above to obtain the matrices $A, B, C$ in the statement of Theorem 2.1, and thus the numbers $\alpha, \beta, \gamma, \delta$ are obtained. Note that we have a $\mathbb{Z}_{2} \times \mathbb{Z}_{2}$ action on $(\alpha, \beta, \gamma, \delta)$ : if we replace $\alpha$ with $-\alpha$, then $a$ and $b$ change sign, but $c$ stays the same. If we then conjugate using $z \mapsto-z$ (which sends figure 1 to its mirror image), we obtain the matrices $-A,-B$ and $C$. Similarly $\delta \mapsto-\delta$ causes $A$ and $C$ to change sign. Thus by insisting that $\alpha, \delta>0$ we obtain not just a triple of elements in $\operatorname{PSL}(2, \mathbb{R})$ but a definite choice of matrices in $S L(2, \mathbb{R})$ representing these Möbius transformations. Having done this, we can read off the sextuple of (signed) traces by using the formula (2.1) for $a$, along with $b=\alpha+1 / \alpha+\beta / \alpha, c=\delta+1 / \delta+\gamma / \delta$, and also $x=(\beta+\gamma+1) / \alpha \delta, y=\delta(x+a)-b$ 
and $z=\alpha(x+a)-c$. But alternatively, once we know this sextuple then we can invert to get back to

$$
\alpha=\frac{z+c}{x+a}, \quad \delta=\frac{y+b}{x+a} \quad \text { and } \quad \beta=\alpha b-1-\alpha^{2}, \gamma=\delta c-1-\delta^{2} .
$$

(Note that if $x+a=0$, then $A B C$ is the identity map, which cannot occur.) Thus if we had $(\alpha, \beta, \gamma, \delta)$ and $\left(\alpha^{\prime}, \beta^{\prime}, \gamma^{\prime}, \delta^{\prime}\right)$ giving rise to matrix triples $(A, B, C)$ and $\left(A^{\prime}, B^{\prime}, C^{\prime}\right)$ representing the same point $p \in \mathcal{T}$, then there would exist a matrix $K$ with $K A K^{-1}= \pm A^{\prime}$ and similarly for $B$ and $C$. Hence the sextuple of traces obtained must be the same up to sign in both cases, and as $A B C$ and $A^{\prime} B^{\prime} C^{\prime}$ both have trace -2 we must have $K A B C K^{-1}=A^{\prime} B^{\prime} C^{\prime}$. Thus we are able to make an even number of sign changes to $A^{\prime}, B^{\prime}, C^{\prime}$ so that now $K A K^{-1}=A^{\prime}$, and the same for $B$ and $C$. As we have seen, this can be achieved merely by changing the signs of $\alpha^{\prime}$ and $\delta^{\prime}$. But now $(\alpha, \beta, \gamma, \delta)$ and $\left( \pm \alpha^{\prime}, \beta^{\prime}, \gamma^{\prime}, \pm \delta^{\prime}\right)$ give rise to the same sextuple, including signs, and given the sextuple we can recover $(\alpha, \beta, \gamma, \delta)$. Thus $(\alpha, \beta, \gamma, \delta)=\left( \pm \alpha^{\prime}, \beta^{\prime}, \gamma^{\prime}, \pm \delta^{\prime}\right)$ and by insisting that $\alpha, \alpha^{\prime}, \delta, \delta^{\prime}>0$, the two are equal.

Now suppose we take any $(\alpha, \beta, \gamma, \delta)$ with $\alpha, \delta>0, \beta, \gamma \neq 0$ and form matrices $B$ and $C$. If $\alpha^{2} \delta^{2} \neq \beta \gamma$, then we can find a unique value of $a$ from (2.1) to obtain $A$ such that $\operatorname{tr}(A B C)=\operatorname{tr}(C B A)=-2$. Of course this may well not be a twicepunctured torus group or even a discrete group. However $(\alpha, \beta, \gamma, \delta)$ will be enough to determine $(A, B, C)$ as a triple in $P S L(2, \mathbb{R})^{3}$ up to simultaneous conjugacy provided all the steps in the uniqueness argument go through here as well. This will happen as long as $k=x+a \neq 0$, so that $A B C \neq-I$.

We now want to determine which $\alpha, \beta, \gamma, \delta$ give rise to a twice-punctured torus group, in order to describe $\mathcal{T}$ exactly. The key result is that as $\mathcal{T}$ is the Teichmüller space of a surface of finite area sitting inside the representation space $\mathcal{R}$ (which is equipped with a topology) then $\mathcal{T}$ must be a connected component of $\mathcal{R}$, as it has no boundary (see [4]). Therefore as soon as we have found a component of $\mathcal{R}$ containing one twice-punctured torus group we are done. Moreover we do not even need to work with the whole of $\mathcal{R}$ (which in general can be non-Hausdorff and so cannot be embedded into $\mathbb{R}^{n}$ ), just a portion of $\mathcal{R}$ which we know contains $\mathcal{T}$, as it will be a connected component of this subset too. Thus from the work so far, we embed $\mathcal{R}$ in $\mathbb{R}^{4}$ (throwing away some points) by defining it to be the set of $(\alpha, \beta, \gamma, \delta)$ such that $\alpha, \delta>0, \beta, \gamma \neq 0$ and so that the triple $(A, B, C)$ of elements of $\operatorname{PSL}(2, \mathbb{R})$ given by the formula in Theorem 2.1 is obtained uniquely up to simultaneous conjugacy. This may seem a strange definition, but we know from Theorem 2.1 that this will be a subspace of the representation space, and it must contain $\mathcal{T}$ somewhere inside (because these groups are certainly obtained by only one $(\alpha, \beta, \gamma, \delta)$ in $\mathcal{R})$, and this is all we need.

Theorem 2.2. The Teichmüller space $\mathcal{T}$ of the twice-punctured torus can be identified with the set

$$
S=\left\{(\alpha, \beta, \gamma, \delta) \in(0, \infty)^{4}: \alpha^{2} \delta^{2}<\beta \gamma\right\}
$$

according to the correspondence in Theorem 2.1.

Proof. The set $S$ itself is clearly connected, being the image of $(0, \infty)^{4}$ under the $\operatorname{map}\left(x_{1}, x_{2}, x_{3}, x_{4}\right) \mapsto\left(x_{1}, x_{2}+\left(x_{1}^{2} x_{4}^{2}\right) / x_{3}, x_{3}, x_{4}\right)$. We need to show that it is contained in $\mathcal{R}$, and is a connected component. By Theorem 2.1, we need to check 
that any point in $S$ cannot give rise to $(A, B, C)$ with $A B C=-I$. If so, then we would find that $a=-x=-(1+\beta+\gamma) / \alpha \delta$, and putting this into (2.1) would leave us with

$$
\left(\alpha^{2} \delta^{2}-\beta \gamma\right)\left(\alpha^{2}+\delta^{2}+\beta+\gamma\right)=\alpha^{2} \delta^{2}+\beta \gamma+\beta \alpha^{2}+\gamma \delta^{2} .
$$

But the left hand side is negative in $S$ whereas the right hand side is positive, so this can never happen and thus $S$ lies inside $\mathcal{R}$. The only points in $\mathcal{R}$ that could be in the closure of $S$ must have $\alpha^{2} \delta^{2}=\beta \gamma$. If this is the case, then we form the matrices $A, B, C$ as above and look to see if $\operatorname{tr}(A B C)$ and $\operatorname{tr}(C B A)$ are -2 . We are fine for $A B C$, but $\operatorname{tr}(C B A)=-2$ precisely when (2.1) is satisfied. Thus we require the right hand side of (2.1) to be zero, obtaining $2 \alpha^{2} \delta^{2}=-\beta \alpha^{2}-\gamma \delta^{2}$. But this can only be obtained if at least one of $\beta, \gamma$ is negative, which are not points that are in the closure of $S$ in $\mathbb{R}^{4}$, let alone $\mathcal{R}$.

We now must show that this is the right component of $\mathcal{R}$. Taking $(\alpha, \beta, \gamma, \delta)=$ $(1,2,2,1)$, we certainly get a point in $S$. From this, we obtain $(a, b, c, x, y, z)=$ $(4,4,4,5,5,5)$, which satisfies all the conditions in Theorem 1.1, and so is a twicepunctured torus group (and, under our normalisation, a subgroup of $\operatorname{PSL}(2, \mathbb{Z})$ of index 12).

So we see how using matrix coordinates enables us to construct twice-punctured torus groups easily and directly, as it is very straightforward to find four positive numbers so that the one condition in Theorem 2.2 is satisfied. Moreover, if anyone else has their preferred normalisation, we can convert efficiently from their coordinates to ours and back again by going via the traces, which are conjugation invariant. Thus in this way, we can easily find the subset that is Teichmüller space in any parametrisation.

\section{TRACE PARAMETERS}

For a two generator group the trace parameters $(a, b, c)=(\operatorname{tr}(A), \operatorname{tr}(B), \operatorname{tr}(A B))$ determine the elements $A$ and $B$ up to simultaneous conjugacy (provided the commutator does not have trace equal to 2), and therefore this is an ideal model for the representation space of any two generator group. Using this for work on the once-punctured torus (see [16]), Minsky asks if there exist similar trace parameters for other surfaces.

For the twice-punctured torus, we can do this. If we are given any three elements $(A, B, C) \in S L(2, \mathbb{R})^{3}$, then we form the sextuple $(a, b, c, x, y, z)$ as defined in the last section. We can then find traces of other elements in $G=\langle A, B, C\rangle$ by using the identity $\operatorname{tr}(X) \operatorname{tr}(Y)=\operatorname{tr}(X Y)+\operatorname{tr}\left(X Y^{-1}\right)$ for all $X, Y \in S L(2, \mathbb{R})$. However, we cannot quite find all traces of words in $A, B, C$ in terms of these six quantities alone, as $s=\operatorname{tr}(A B C)$ and $t=\operatorname{tr}(C B A)$ can only be written as roots of the same quadratic, and therefore cannot be distinguished. In fact by expanding the expression $\operatorname{tr}(A B C)+\operatorname{tr}\left(A^{-1} B C\right)$, we obtain an expression for the sum of these traces:

$$
s+t=a x+b y+c z-a b c
$$

and by considering $\operatorname{tr}(A B C) \operatorname{tr}\left(C^{-1} A^{-1} B^{-1}\right)$ we find

$$
s t=x y z+a^{2}+b^{2}+c^{2}+x^{2}+y^{2}+z^{2}-a b z-b c x-c a y-4 .
$$

(These equations, which are sometimes referred to as Fricke's Lemma, date back even earlier to the end of the last century, see [20] and [10].) Thus it may be possible 
to have two triples in $S L(2, \mathbb{C})^{3}$, say $(A, B, C)$ and $\left(A^{\prime}, B^{\prime}, C^{\prime}\right)$, giving rise to the same sextuple of real numbers but which are not simultaneously conjugate. To see this can occur, we construct a continuum of examples by making $B$ a diagonal matrix and $A$ non-diagonal, say

$$
A=\left(\begin{array}{cc}
2 & 1 \\
1 & 1
\end{array}\right), \quad B=\left(\begin{array}{cc}
2 & 0 \\
0 & \frac{1}{2}
\end{array}\right) .
$$

Then by setting $C=K A K^{-1}$, where $K$ commutes with $B$ but not $A$, we must have $a=c$ and $x=z$. Thus the sextuple of traces remains invariant by swapping $A$ and $C$. By direct calculation, we find that $\operatorname{tr}(A B C)=17 / 2+2 \lambda^{2}+1 /\left(2 \lambda^{2}\right)$ and $\operatorname{tr}(C B A)=17 / 2+\lambda^{2} / 2+2 / \lambda^{2}$, where $K$ has diagonal $(\lambda, 1 / \lambda)$. This means that $(A, B, C)$ and $(C, B, A)$ are not simultaneously conjugate unless $\lambda$ is a cube root of unity.

Thus this sextuple of traces cannot be a general global parametrisation for triples of elements of $P S L(2, \mathbb{R})$. Moreover, we cannot obtain a global parametrisation for $n$-tuples of elements of $\operatorname{PSL}(2, \mathbb{R})$ up to conjugacy when $n \geq 3$ by using traces of words of length one or two in the generating elements, because the automorphism of the free group $F_{n}$ given by sending each element to its inverse will preserve all such traces but will in general produce a non-conjugate $n$-tuple. (In the case $n=2$, this automorphism can always be induced by conjugation in $P G L(2, \mathbb{R})$ provided that the trace of the commutator is not equal to 2.) However in our case we know that $s, t=-2$, and so the two equations above must be satisfied. Provided we stick to sextuples giving rise to a twice-punctured torus group, then these six variables subject to two relations (and appropriate sign choices, say $a, b>0$ ) do determine our triple up to conjugacy, because there will be a unique point $(\alpha, \beta, \gamma, \delta) \in \mathcal{T}$ giving rise to a triple with these traces. Thus this variety in $\mathbb{R}^{6}$ given by two equations has a component which is Teichmüller space, which we can describe (though not neatly) using Theorem 2.2, by writing $\beta \gamma>\alpha^{2} \delta^{2}$ in terms of the traces, as given by the formulae for the traces in the proof of Theorem 2.1. This component is therefore exactly the same set as that obtained from Theorem 1.1, despite the different equations. But one difficulty with this approach is that it is harder to explicitly write down points in this description of Teichmüller space because of the two relations, and the various inequalities that need to be satisfied. For instance, try to find directly a sextet of rational (or integral) numbers satisfying the above. However, with the matrix parameters there are no equations to be satisfied amongst them and only one inequality, so we can write down points in Teichmüller space immediately.

\section{REFERENCES}

[1] L. V. Ahlfors, On Quasiconformal mappings, J. Analyse Math. 3 (1954), 1-58. MR 16:348d

[2] L. Bers, On boundaries of Teichmüller spaces and on Kleinian Groups. I, Ann. of Math. 91 (1970), 570-600. MR 45:7044

[3] L. Bers, Finite dimensional Teichmüller spaces and generalisations, Bull. Amer. Math. Soc. 5 (1981), 131-172. MR 82k:32050

[4] J. O. Button, Teichmüller spaces, Schottky groups and representations of surface groups, Preprint, University of Oxford (1999).

[5] C. Earle, Some intrinsic coordinates on Teichmüller space, Proc. Amer. Math. Soc. 83 (1981), 527-531. MR 82j:32050

[6] A. Fathi, F. Laudenbach and V. Poenaru, Travaux de Thurston sur les surfaces, Asterique 66-67 (1979). MR 82m:57003 
[7] L. Keen, Intrinsic moduli on Riemann Surfaces, Ann. of Math. 84 (1966), 404-420. MR 34:2859

[8] L. Keen, On Fricke Moduli, Advances in the Theory of Riemann surfaces (L. Ahlfors et al., eds.), Ann. Math. Studies 66 (1971), Princeton University Press, Princeton, New Jersey. MR 44:5450

[9] I. Kra, Horocyclic coordinates for Riemann surfaces and Moduli spaces. I: Teichmüller and Riemann spaces of Kleinian groups, J. Amer. Math. Soc. 3 (1990), 499-578. MR 91c:32014

[10] W. Magnus, Rings of Fricke Characters and Automorphism Groups of Free Groups, Math. Z. 170 (1980), 91-103. MR 81a:20043

[11] B. Maskit, Moduli of marked Riemann surfaces, Bull. Amer. Math. Soc. 80 (1974), 773-777. MR 49:10875

[12] B. Maskit, Parameters for Fuchsian groups. I: Signature (0,4). Holomorphic functions and moduli, Vol. II (D. Drasin et al., eds.), Math. Sci. Res. Inst. Publ. 11 (1988), 251-265. MR 90g:30042

[13] B. Maskit, Parameters for Fuchsian groups. II: Topological type (1,1), Ann. Acad. Sci. Fenn. Ser. A I Math. 14 (1989), 265-275. MR 91a:30043

[14] B. Maskit, Explicit matrices for Fuchsian groups, Contemp. Math. Amer. Math. Soc. 169 (1994), 451-466. MR 96f:30045

[15] B. Maskit, New parameters for Fuchsian groups of genus 2, Proc. Amer. Math. Soc. 127 (1999), 3643-3652. MR 2000b:32031

[16] Y. N. Minsky, The classification of punctured-torus groups, Ann. of Math. 149 (1999), 559626. MR 2000f:30028

[17] M. Seppälä and T. Sorvali, Parametrisation of Möbius groups acting in a disc, Comment. Math. Helv. 61 (1986), 149-160. MR 87i:20089

[18] M. Seppälä and T. Sorvali, Parametrisation of Teichmüller spaces by geodesic length functions, Holomorphic functions and moduli, Vol. II (D. Drasin et al., eds.), Math. Sci. Res. Inst. Publ. 11 (1988), 267-284. MR 89m:32040

[19] W. Thurston, Three-Dimensional Geometry and Topology. Volume 1, Princeton University Press, Princeton, New Jersey (1997). MR 97m:57016

[20] H. Vogt, Sur les invariants, fondamentaux des équations différentielles linéaires du second ordre, Ann. Sci. Ecole. Norm. Sup. (3) 6 (1889), Suppl. 3-72.

Wadham College, University of Oxford, OX1 3PN, England, United Kingdom

E-mail address: button@maths.ox.ac.uk 